\title{
A new approach to the r-Whitney numbers by using combinatorial differential calculus
}

\author{
Miguel A. Méndez \\ Departamento de Matemáticas, \\ Facultad de Ciencias, \\ Universidad Antonio Nariño, \\ Bogotá, Colombia \\ email: mmendezenator@gmail.com
}

\author{
José L. Ramírez \\ Departamento de Matemáticas, \\ Universidad Nacional de Colombia, \\ Bogotá, Colombia \\ email: jlramirezr@unal.edu.co
}

\begin{abstract}
In the present article we introduce two new combinatorial interpretations of the $r$-Whitney numbers of the second kind obtained from the combinatorics of the differential operators associated to the grammar $\mathrm{G}:=\left\{\mathrm{y} \rightarrow \mathrm{y} \mathrm{x}^{\mathrm{m}}, \mathrm{x} \rightarrow \mathrm{x}\right\}$. By specializing $\mathrm{m}=1$ we obtain also a new combinatorial interpretation of the $r$-Stirling numbers of the second kind. Again, by specializing to the case $r=0$ we introduce a new generalization of the Stirling number of the second kind and through them a binomial type family of polynomials that generalizes Touchard's polynomials. Moreover, we recover several known identities involving the $r$-Dowling polynomials and the $r$-Whitney numbers using the combinatorial differential calculus. We construct a family of posets that generalize the classical Dowling lattices. The $r$-Withney numbers of the first kind are obtained as the sum of the Möbius function over elements of a given rank. Finally, we prove that the r-Dowling polynomials are a Sheffer family relative to the generalized Touchard binomial family, study their umbral inverses, and introduce [m]-Stirling numbers of the first kind. From the relation between umbral calculus and the Riordan matrices we give several new combinatorial identities
\end{abstract}

2010 Mathematics Subject Classification: 11B83, 11B73, 05A15, 05A19

Key words and phrases: differential operators, r-Whitney number, r-Dowling polynomial 


\section{Introduction}

The $r$-Whitney numbers of the second kind $W_{m, r}(n, k)$ were defined by Mező $[24]$ as the connecting coefficients between some particular polynomials. For non-negative integers $n, k$ and $r$ with $n \geq k \geq 0$ and for any integer $m>0$

$$
(m x+r)^{n}=\sum_{k=0}^{n} m^{k} W_{m, r}(n, k) x^{\underline{k}},
$$

where $x^{\underline{n}}=x(x-1) \cdots(x-\mathfrak{n}+1)$ for $\mathfrak{n} \geq 1$ and $x^{\underline{0}}=1$.

Cheon and Jung [8] showed that the numbers $W_{m, r}(n, k)$ are related to the Dowling lattices as follows. Let $\mathrm{Q}_{\mathfrak{n}}(\mathrm{G})$ be the Dowling lattice of rank $\mathrm{n}$, where $G$ is a finite group of order $m$. The coefficient of $r^{s}$ of the polynomial $\sum_{k=0}^{n} W_{m, r}(n, k)$ is equal to the number of elements of $Q_{n}(G)$ containing $n-s$ distinct unit functions. This sequence generalizes the Whitney numbers of the second kind, $W_{m}(n, k)=W_{m, 1}(n, k)$, that count the total number of elements of corank $k$ in $Q_{n}(G)[11]$.

Mihoubi and Rahmani [27] found an interesting combinatorial interpretation for the $r$-Whitney numbers of the second kind by using colored set partitions. Recall that a partition of a set $A$ is a class of disjoint subsets of $A$ such that the union of them covers $A$. The subsets are called blocks. Let $r, n \geq 0$ be integers, and let $A_{n, r}$ be the set defined by $A_{n, r}:=\{1,2, \ldots, r, r+1, \ldots, n+r\}$. The elements $1,2, \ldots, r$ will be called special elements. A block of a partition of the above set is called special if it contains special elements. Then $W_{m, r}(n, k)$ counts the number of the set partitions of $A_{n, r}$ in $k+r$ blocks, such that the elements $1,2, \ldots, r$ are in distinct blocks (i.e., any special block contains exactly one special element). All the elements but the last one in non-special blocks are coloured with one of $m$ colours independently and neither the elements in the special blocks nor the special blocks are coloured.

The r-Whitney numbers satisfy the following recurrence relation [24]

$$
W_{m, r}(n, k)=W_{m, r}(n-1, k-1)+(k m+r) W_{m, r}(n-1, k) .
$$

Moreover, these numbers have the exponential generating function [24]:

$$
\sum_{n=k}^{\infty} W_{m, r}(n, k) \frac{z^{n}}{n !}=\frac{e^{r z}}{k !}\left(\frac{e^{m z}-1}{m}\right)^{k} .
$$

Note that if $(m, r)=(1,0)$ we obtain the Stirling numbers of the second kind, if $(m, r)=(1, r)$ we have the $r$-Stirling (or noncentral Stirling) numbers 
[4], and if $(m, r)=(m, 1)$ we have the Whitney numbers [1, 2]. Many properties of the $r$-Whitney numbers and their connections to elementary symmetric functions, matrix theory, special polynomials, combinatorial identities and generalizations can be found in $[9,10,12,18,20,21,24,25,26,28,29,37]$.

The combinatorial differential calculus was introduced by Joyal in the framework of combinatorial species (see for example [15, 3, 16]). However, by using directly exponential formal power series and the combinatorics of their coefficients (see [19]), the fundamentals of the approach can be explained without the use of the categorical framework involved in the theory of species. The combinatorial differential calculus is closely related to Chen context-free grammar method [6]. However, they are not equivalent. Differential operators that are not derivations can be combinatorially interpreted, and algebraic formulas obtained from that interpretation. Only derivations have a counterpart in the context of Chen grammars. The substitution rules in a context-free grammar can be translated into a differential operator. By the iterated application of the combinatorial version of it, the associated combinatorial objects emerge, and not infrequently, in a simple and natural way.

Using the combinatorial differential calculus we present a new combinatorial interpretation to the r-Whitney numbers of the second kind. As a special case we get a new combinatorial interpretation for the r-Stirling numbers of the second kind and introduce the [m]-Stirling numbers of the second kind, $S^{[m]}(n, k)$. Through them we define the [m]-Touchard polynomials, that generalize the classical family and are also of binomial type. Their umbral inverse give us the $[\mathrm{m}]$-Stirling numbers of the first kind. The combinatorial interpretation of the $r$-Whitney numbers (and of the generalization of the Stirling numbers) of the second kind we present here are very natural. We construct a family of posets $Q_{n, r}(G), G$ a group of order $m$, that generalize the Dowling lattices. Our interpretation of $r$-Withney numbers directly count the number of elements of a given rank on those posets, and by Möbius inversion we get the $r$-Whitney numbers of the first kind, as the sum of their Möbius function evaluated at elements of a given rank (Theorem 2 bellow). The $r$-Stirling numbers of the first and second kind are interpreted in a similar way by specializing $G$ to the trivial group $G=\{e\}$. Also a similar interpretation is given in terms of a subposet $Q_{n, 0}(G)$ of $Q_{n, r}(G)$ for the $[m]$-Stirling numbers (see Remark 2).

By using classical results of umbral calculus we get that the $r$-Dowling polynomials are of Sheffer type relative to the $[\mathrm{m}]$-Touchard binomial family. Finally, from the relation between umbral calculus and the Riordan matrices we give several new combinatorial identities involving the $r$-Whitney number of both kinds, Bernoulli polynomials and Euler polynomials. 


\section{New combinatorial models for $r$-Whitney, r-Stirling, and new [m]-Stirling numbers}

Chen [6] introduced a combinatorial method by means of context-free grammar to study exponential structures. A context-free grammar $\mathrm{G}$ over an alphabet $X$, whose symbols are commutative indeterminates, is a set of productions or substitutions rules that replace a symbol of $X$ by a formal function (formal power series) in the set of indeterminates $X, x \rightarrow \phi_{x}(X), x \in A$.

Here $A$ is a subset of the alphabet $X$. The formal derivative $D$ is a linear operator defined with respect to a context-free grammar $G$ such that for a letter $x \in A$ acts by substitution by $\phi(X)$, and it is extended recursively as a derivation. For any formal functions $u$ and $v$ we have:

$D(u+v)=D(u)+D(v), \quad D(u v)=D(u) v+u D(v), \quad D(f(u))=\frac{\partial f(u)}{\partial u} D(u)$

where $f(x)$ is a formal power series. For more applications of the context-free grammar method see for example [5, 7, 13, 17].

The grammar formal derivative $\mathrm{D}$ can be equivalently written as a derivation in the algebra of formal power series over a ring $\mathcal{R}$ containing $\mathbb{Q}, \mathcal{R}[[\mathrm{X}]]$.

$$
\mathcal{D}=\sum_{x \in A} \phi_{x}(X) \partial_{x}
$$

For $A$ infinite, we have to make a summability assumption over the family of formal power series $\left\{\phi_{x}(X)\right\}_{x \in A}$. The variables in $X$ are thought of as colors. Each operator $\phi_{x}(X) \partial_{x}$ is combinatorially interpreted as a corolla having as root a ghost vertex of color $x$ and weighted with the coefficients of the series $\phi(X)$ according with the distribution of colors of the leaves in the corolla. A formal power series $F(X)$ are also represented by corollas in a similar way but without the ghost vertex. The combinatorial representation of the operator acting over a series $F(X)$ is obtained by dropping over the combinatorial representation of $F(X)$ corollas whose ghost vertices replace vertices of the same color on corollas representing F (see Figure 1). Details and proofs of why this combinatorial approach works can be seen in [19].

As an example let us consider the Stirling grammar ([6])

$$
G:=\left\{\begin{array}{l}
y \rightarrow x y \\
x \rightarrow x .
\end{array}\right.
$$



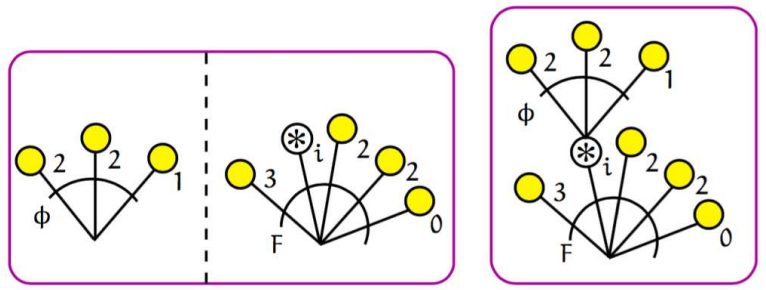

Figure 1: Corolla operator $\phi(X) \partial_{i}$ applied to $F$.

Applying the associated formal operator $\mathfrak{n}$ times to $y$, it is known that

$$
D^{n} y=\sum_{k=1}^{n} S(n, k) x^{k} y
$$

where $S(n, k)$ are the Stirling numbers of the second kind. The differential operator associated to that grammar is $\mathcal{D}=x y \partial_{y}+x \partial_{x}$. We call it the Stirling operator. The variables $x$ and $y$ are represented by vertices in two colors, yellow and white. The operator $\mathcal{D}$ has as combinatorial representation the sum of two corollas. The operator $x y \partial_{y}$ acts over a white vertex replacing it by a 'ghost' vertex $*$ and connecting it to a yellow vertex $x$ and to white one $y$. The operator $x \partial_{x}$ places a ghost vertex in a yellow vertex and connects it to another yellow vertex (see Figure 2).

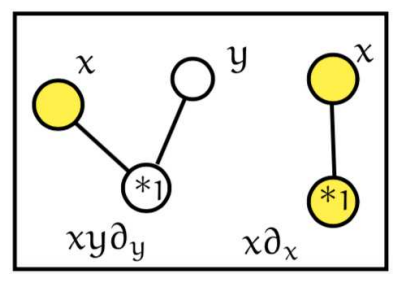

Figure 2: Combinatorial operator $\mathcal{D}=x y \partial_{y}+x \partial_{x}$.

When applying the operator more than one time, for simplicity, we replace the ghost vertices by numbers to keep track of the order in which the operator was applied. If we apply it $\boldsymbol{n}$ times to $y$ (combinatorially represented as a singleton vertex of color white) we obtain an increasing tree with a path (spine) from the root to the white vertex on the top. Along each node of the spine 
there are linear branches having in the top of each of them one yellow vertex with weight $y$. The elements of the branches along the spine form a partition $\pi$ of $[n]$. The weight of each such tree is equal to $x$ to the number of branches (blocks of $\pi$ ) times $y$, the weight of the white vertex on top of the spine (see Figure 3). In this way we get a visual proof of Equation (2).

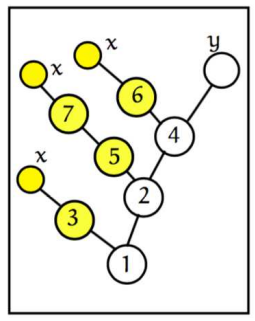

Figure 3: A tree enumerate by $\mathcal{D}^{7} y=\left(x y \partial_{y}+x \partial_{x}\right)^{7} y$ that corresponds to the partition $\pi=\{\{1,3\}\{2,5,7\}\{4,6\}\}$.

Let us now consider the context-free grammar $\mathrm{G}$ defined in [13].

$$
G:=\left\{\begin{array}{l}
y \rightarrow y x^{m} \\
x \rightarrow x .
\end{array}\right.
$$

Hao et al. [13] proved as a particular case that

$$
D^{n} y x=\sum_{k=0}^{n} W_{m}(n, k) y x^{m k+1} .
$$

This grammar $G$ corresponds to the differential operator $\mathcal{D}=y x^{m} \partial_{y}+x \partial_{x}$.

In Figure 4 we show the combinatorial interpretation of this operator for $\mathrm{m}=2$. Moreover, by the main theorem of [13] we have

$$
\mathcal{D}^{n} y x^{r}=\sum_{k=0}^{n} W_{m, r}(n, k) y x^{m k+r} .
$$

In Figures 5 and 6 , we show the first and second derivative for $\mathrm{m}=2$ and $r=3$, respectively.

By the iterated application of the combinatorial version of the operator $\mathcal{D}$, dropping the corollas over one white vertex and $r$ yellow vertices $\left(y x^{r}\right)$ we obtain the following combinatorial structure. A forest consisting of one 


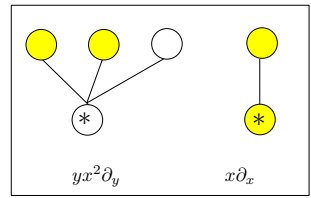

Figure 4: Combinatorial interpretation for the operator $\mathcal{D}=y x^{m} \partial_{y}+x \partial_{x}$, with $\mathfrak{m}=2$.

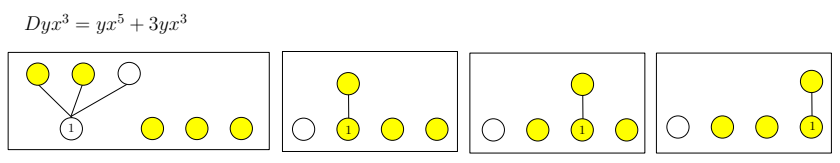

Figure 5: The first derivative.

increasing tree, grown from the initial white vertex $y$, followed by $r$ linearly ordered branchless increasing trees (grown from the $r$ yellow initial vertices, $x^{r}$ ). The trees of this forest are characterized as follows (see Figure 7).

1. The first tree has a spine of white vertices with an unlabeled white vertex at the top having weight $y$. There are $m$ (totally ordered) branches of yellow vertices that sprout from each vertex on the spine. Each branch has an unlabeled yellow vertex at the top, with weight $x$.

2. Each of the $r$ branchless increasing trees consists of a set of internal vertices (that may be empty) with an unlabeled yellow vertex at the top, having weight $x$.

The total weight of each of this forests is equal to $y x^{m k+r}$, where $k$ is the number of vertices in the spine. Then, from Equation (3) we have that the $r$-Whitney numbers $W_{m, r}(n, k)$ count the number of forests as above, having $k$ vertices in the spine, and $n$ internal vertices in total.

Reinterpreting the forests of increasing trees we obtain the following combinatorial interpretation of $W_{m, r}(n, k)$.

Theorem 1 The $r$-Whitney numbers $W_{m, r}(n, k)$ count pairs of the form $\left(\left\{\left(\mathrm{B}, \mathrm{f}_{\mathrm{B}}\right)\right\}_{\mathrm{B} \in \pi}, \mathbf{V}\right)$ where

1. The first component is a partial partition of $\{1,2, \ldots, n\}$, having exactly $k$ blocks, $\uplus_{B \in \pi} B=A \subseteq\{1,2, \ldots, n\}$, plus a coloring on each block, $f_{B}: B \rightarrow[m]$. The coloring on $f_{B}$ assign to the minimum element of $B$ the color 1 . 


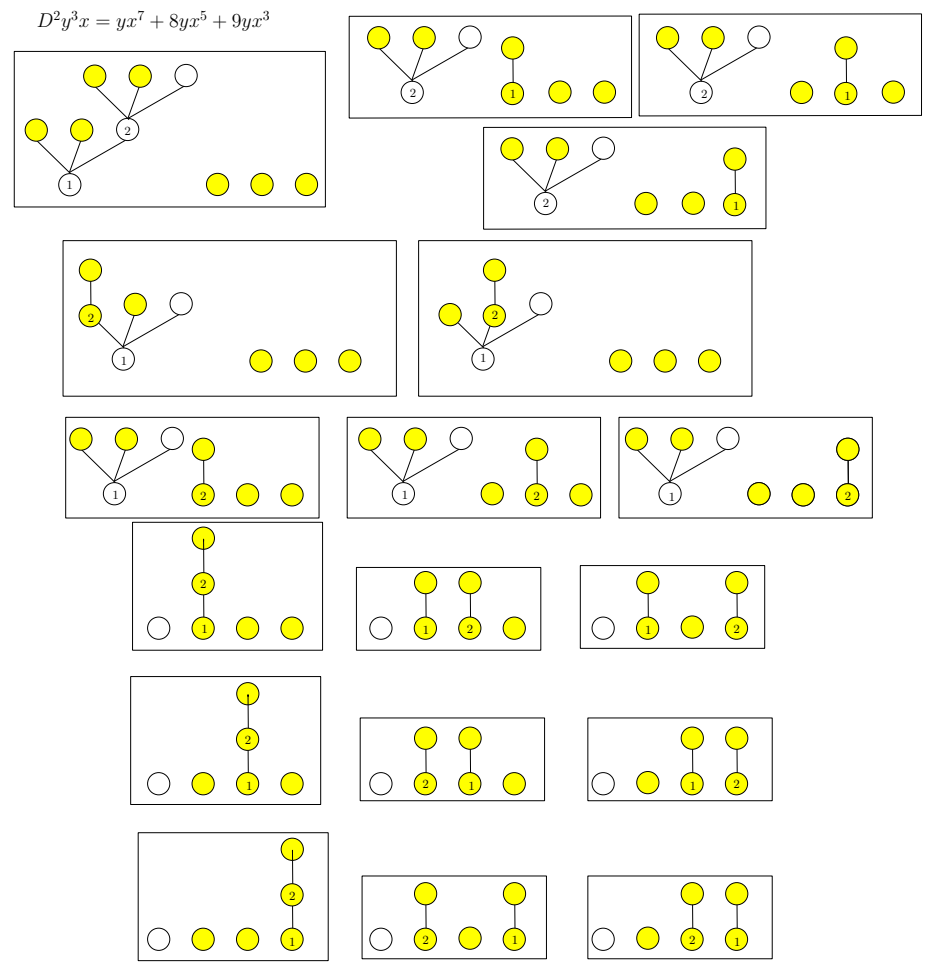

Figure 6: The second derivative.

2. The second component $\mathbf{V}=\left(\mathrm{B}_{1}, \mathrm{~B}_{2}, \ldots, \mathrm{B}_{\mathrm{r}}\right)$ is a weak $\mathrm{r}$-composition of $[n]-A$

$$
\biguplus_{j=1}^{r} B_{r}=[n]-A .
$$

Proof. We are going to prove that the first tree is in bijection with the colored partitions described in Item (1). We assign to each vertex of the spine of a given tree the set of vertices of the $m$ branches attached to it plus the vertex itself. In this way we obtain the partial partition $\pi$ with $k$ blocks in total. Then color the vertices on the ith branch of each block with color $i, i=1,2, \ldots, m$, and assign color 1 to the vertex on the spine. Observe that, since the tree is increasing, the vertex on the spine has the minimum label of its block. This construction is clearly reversible (see Figure 7 ). The $r$ branchless trees are naturally associated to the composition $\mathbf{V}$ by assigning the ith tree its set of internal vertices $B_{i}$. 


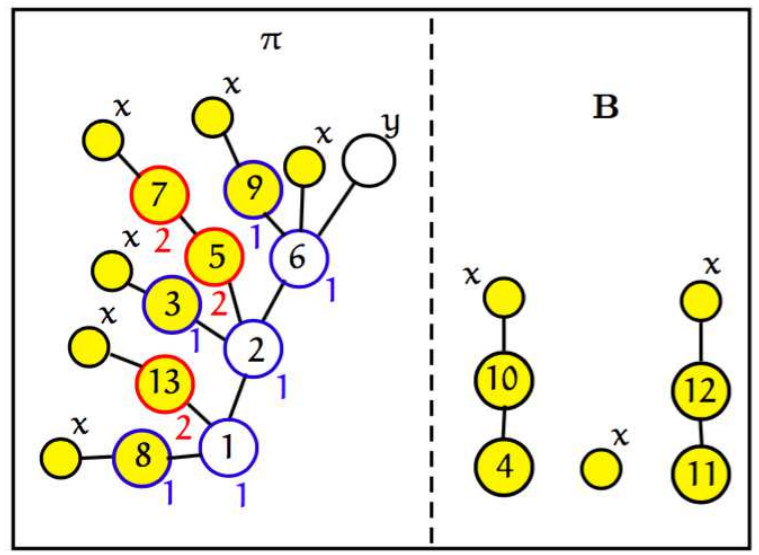

Figure 7: Whitney forest for $m=2$ and $r=3$.

We represent a colored set $\left(B, f_{B}\right)$ by placing the color of each element as its exponent $\left(B, f_{B}\right) \equiv\left\{b^{f(b)} \mid b \in B\right\}$. In this way, the colored partition associated to the tree in Figure 7 is $\left\{\left\{1^{1}, 8^{1}, 13^{2}\right\},\left\{2^{1}, 3^{1}, 5^{2}, 7^{2}\right\},\left\{6^{1}, 9^{1}\right\}\right\}$. The composition is equal to $\mathbf{V}=(\{4,10\}, \emptyset,\{11,12\})$.

Example 1 The $r$-Whitney number $\mathrm{W}_{2,2}(2,2)=1$, the pair being $\left(\left\{\left\{1^{1}\right\},\left\{2^{1}\right\}\right\},(\emptyset, \emptyset)\right)$. For $W_{2,3}(2,1)=8$, it enumerates pairs, the first component is a 2-colored partition on a subset $\mathrm{A}$ of $\{1,2\}$ having one block. The second component a weak 3-composition of [2] - A. The pairs being
1. $\left(\left\{\left\{1^{1}, 2^{1}\right\}\right\},(\emptyset, \emptyset, \emptyset)\right)$,
2. $\left(\left\{\left\{1^{1}, 2^{2}\right\}\right\},(\emptyset, \emptyset, \emptyset)\right)$,
3. $(\{\{1\}\}\},(\{2\}, \emptyset, \emptyset))$,
4. $\left(\left\{\left\{1^{1}\right\}\right\},(\emptyset,\{2\}, \emptyset)\right)$,
5. $\left(\left\{\left\{1^{1}\right\}\right\},(\emptyset, \emptyset,\{2\})\right)$,
6. $\left(\left\{\left\{2^{1}\right\}\right\},(\{1\}, \emptyset, \emptyset)\right)$,
7. $\left(\left\{\left\{2^{1}\right\}\right\},(\emptyset,\{1\}, \emptyset)\right)$,
8. $\left(\left\{\left\{2^{1}\right\}\right\},(\emptyset, \emptyset,\{1\})\right)$. 
Remark 1 We get the following combinatorial interpretation for the generalized Stirling numbers of the second kind obtained by specializing $\mathrm{r}$ and $\mathrm{m}$.

1. For $\mathrm{m}=1$ we get a combinatorial interpretation for the $\mathrm{r}$-Stirling numbers of the second kind $S_{r}(n, k)$. They count the pairs $(\pi, \mathbf{V}), \pi$ a partition of some subset $\mathrm{A}$ of $[\mathrm{n}], \mathbf{V}$ a weak $\mathbf{r}$-composition of $[\mathrm{n}]-\mathrm{A}$.

2. For $\mathrm{r}=0$ we get a new generalization $\mathrm{S}^{[\mathrm{m}]}(\mathrm{n}, \mathrm{k})$ of the Stirling numbers of the second kind. It counts the number of colored partitions, as in Theorem 1, Item (1), but over the whole set [n].

Definition 1 We define the $[\mathrm{m}]$-Touchard polynomials, $\mathrm{T}_{\mathrm{n}}^{[\mathrm{m}]}(\mathrm{x})$, by

$$
\mathrm{T}_{\mathrm{n}}^{[\mathrm{m}]}(\mathrm{x}):=\sum_{\mathrm{k}=1}^{\mathrm{n}} \mathrm{S}^{[\mathrm{m}]}(\mathrm{n}, \mathrm{k}) \mathrm{x}^{\mathrm{k}} .
$$

The polynomial family $\left\{\mathrm{T}_{\mathfrak{n}}^{[\mathrm{m}]}(\mathrm{x})\right\}_{\mathfrak{n}=0}^{\infty}$ is of binomial type, i.e.,

$$
\begin{aligned}
T_{0}^{[m]}(x) & =1 \\
T_{n}^{[m]}(x+y) & =\sum_{k=0}^{n}\left(\begin{array}{l}
n \\
k
\end{array}\right) T_{k}^{[m]}(x) T_{n-k}^{[m]}(y) .
\end{aligned}
$$

We shall prove it in Section 5.

\section{The r-Dowling posets}

Let $B_{1}$ and $B_{2}$ be two disjoint sets, and $f_{i}$ two functions, $f_{i}: B_{i} \rightarrow C, i=1,2$. Recall that the disjoint union of $f_{1}$ and $f_{2}, f_{1} \uplus f_{2}: B B_{1} \uplus B_{2} \rightarrow C$ is defined as follows:

$$
f_{1} \uplus f_{2}(x)= \begin{cases}f_{1}(x), & \text { if } x \in B_{1} ; \\ f_{2}(x), & \text { if } x \in B_{2} .\end{cases}
$$

Let $\mathrm{G}$ be a finite group of order $\mathrm{m}$. We consider the set of pairs as in Theorem 1 , but where each function $f_{B}, B \in \pi$, colors the elements of the block $B$ with elements of the group $G$ (instead of $[\mathrm{m}]$ ) in such a way that it assigns to the minimum element of $B$ the identity of $G$. We call that kind of colorations unital. Consider functions $f:[n] \rightarrow G$ of the form $f=\uplus_{B \in \pi} f_{B}$, where each coloring $f_{B}: B \rightarrow G$ is unital. By simplicity, the pair $(\pi, f)$ will be denoted by $\pi^{f}$ and will be called a (unital) colored partition. Let $Q_{n, r}(G, k)$ be the set of 
unital colored partitions-composition pairs on [n], where the colored partition has exactly $k$ blocks. Denote by $Q_{n, r}(G)$ the union $\uplus_{k=0}^{n} Q_{n, r}(G, k)$, i.e., the set of colored partition-composition pairs $\left(\pi^{f}, \mathbf{V}\right)$ having $\pi$ an arbitrary number of blocks, $k=0,1,2, \ldots, n$. Clearly, by Theorem 1 , the number of unital colored partition-composition pairs in $Q_{n, r}(G, k)$ is the $r$-Whitney number $W_{m, r}(n, k)$, that is $W_{m, r}(n, k)=\left|Q_{n, r}(G, k)\right|$. We are going to define a partial order on $\mathrm{Q}_{n, r}(\mathrm{G})$ such that its ranked Möbius function gives us the $r$-Whitney numbers of the first kind,

$$
w_{\mathrm{m}, \mathrm{r}}(\mathrm{n}, \mathrm{k})=\sum_{\left(\pi^{\mathrm{f}}, \mathbf{V}\right),|\pi|=k} \mu\left(\widehat{0},\left(\pi^{\mathrm{f}}, \mathbf{V}\right)\right) .
$$

This construction generalizes the classical Dowling lattice [11], recovered as a particular case of this by making $r=1$.

For a coloration $f: A \rightarrow G$, and an element $g$ of the group, we define a new coloration $\mathrm{f} * \mathrm{~g}$ by

$$
(f * g)(x):=f(x) * g, x \in A,
$$

where $*$ is the operation of the group.

We introduce two kind of operations on the set $Q_{n, r}(G)$. Let $\left(\pi^{f}, \mathbf{V}\right) \in$ $\mathrm{Q}_{\mathrm{n}, \mathrm{r}}(\mathrm{G})$. We obtain another colored partition-composition pair $\left(\tau^{\mathrm{h}}, \mathbf{V}^{\prime}\right) \in$ $\mathrm{Q}_{\mathrm{n}, \mathrm{r}}(\mathrm{G})$ by the following two kinds of operations.

1. The compositions remain unchanged, i.e., $\mathbf{V}=\mathbf{V}^{\prime}$, and $\tau$ is obtained from $\pi$ by joining some blocks of $\pi, B_{1}, B_{2}, \ldots, B_{\ell}, B=\uplus_{i=1}^{\ell} B_{i}$ (we assume that the family is listed according with the minimum element of the blocks; $\left.\min B_{i}<\min B_{i+1}\right)$. The rest of blocks remain the same. The coloring $\mathrm{h}$ is obtained in the block $\mathrm{B}$ as a unital linear combination

$$
h_{B}=f_{B_{1}} * g_{1} \uplus f_{B_{2}} * g_{2} \uplus \cdots \uplus f_{B_{\ell}} * g_{\ell},
$$

where $g_{1}=e$ is the identity of $G$, and $g_{2}, g_{3}, \ldots, g_{\ell}$ are arbitrary elements of $\mathrm{G}$. The coloring on the rest of the blocks of $\tau$ remain the same, $h_{B^{\prime}}=$ $f_{B^{\prime}}$, for $B^{\prime} \neq B$. It is clear that $h_{B}$ is again unital.

2. The components of $\mathbf{V}^{\prime}$ are the same as the components of $\mathbf{V}$ except one, say $V_{j}$, which is augmented by some blocks of $\pi$; while $\pi$ is reduced by these blocks. Precisely:

$$
\begin{aligned}
V_{i}^{\prime} & =V_{i}, \text { for } i \neq j, \text { and } V_{j}^{\prime}=V_{j} \cup \bigcup_{i=1}^{\ell} B_{i}, \\
\pi^{\prime} & =\pi-\left\{B_{1}, B_{2}, \ldots, B_{\ell}\right\}
\end{aligned}
$$


From now on we follow the convention, for concrete examples, of separating the colored partition-composition pair $\left(\pi^{\mathrm{f}}, \mathbf{V}\right)$ with double bars,

$$
\pi^{\mathrm{f}} \| \mathbf{V}:=\left(\pi^{\mathrm{f}}, \mathbf{V}\right)
$$

A colored partition $\pi^{f}$ can be represented as a factored monomial with exponents in G

$$
\pi^{f} \equiv \prod_{B \in \pi}\left(\prod_{b \in B_{i}} b^{f(b)}\right) .
$$

For example, if $\mathrm{G}=\mathrm{F}_{5}^{\times}$is the multiplicative group of the Galois field $\mathrm{F}_{5}=$ $\mathbb{Z} /(5)$, the colored partition $\left\{\left\{1^{1}, 8^{3}, 13^{2}\right\},\left\{2^{1}, 3^{4}, 5^{3}, 7^{2}\right\},\left\{6^{1}, 9^{3}\right\}\right\}$ is represented as the factored monomial $\left(1^{1} 8^{3} 13^{2}\right)\left(2^{1} 3^{4} 5^{3} 7^{2}\right)\left(6^{1} 9^{3}\right)$. A unital linear combination as in Equation (5) can be represented as the expansion of a monomial of monomials,

$$
\prod_{b \in B} b^{h(b)}=\prod_{i=1}^{\ell}\left(\prod_{b \in B_{i}} b^{f_{B_{i}}(b)}\right)^{g_{i}}=\prod_{b \in B} b^{\uplus_{i=1}^{\ell} f_{B_{i}}(b) * g_{i}} .
$$

For example, $1^{1} 8^{3} 13^{2} 2^{2} 3^{3} 5^{1} 7^{4} 6^{4} 9^{2}=\left(1^{1} 8^{3} 13^{2}\right)^{1}\left(2^{1} 3^{4} 5^{3} 7^{2}\right)^{2}\left(6^{1} 9^{3}\right)^{4}$ represents the unital linear combination $g_{1}=1, g_{2}=2, g_{3}=1$ and $g_{4}=2$, with $B_{1}=\{1\}, B_{2}=\{2,3,5,7\}, B_{3}=\{6,9\}, B_{4}=\{8,13\}$. The operations (1) and (2) are clearly closed on $Q_{n, r}(G)$. Now we can define the partial order on $Q_{n, r}(G)$.

Definition 2 Let $\left(\pi^{\mathrm{f}}, \mathbf{V}\right)$ and $\left(\tau^{\mathrm{h}}, \mathbf{V}^{\prime}\right)$ be two elements of $\mathrm{Q}_{\mathrm{n}, \mathrm{r}}(\mathrm{G})$. We say that

$$
\left(\pi^{\mathrm{f}}, \mathbf{V}\right) \leq\left(\tau^{\mathrm{h}}, \mathbf{V}^{\prime}\right)
$$

if $\left(\tau^{\mathrm{h}}, \mathbf{V}^{\prime}\right)$ can be obtained from $\left(\pi^{\mathrm{f}}, \mathbf{V}\right)$ by any sequence of operations (1) or (2) above. It includes de empty sequence, that gives us $\left(\pi^{\mathrm{f}}, \mathbf{V}\right)=\left(\tau^{\mathrm{h}}, \mathbf{V}^{\prime}\right)$. In other words, $\leq$ is defined to be the transitive and reflexive clousure of the union of the binary relations defined by operations (1) and (2).

The resulting relation is antisymmetric because the operations (1) and (2) are directed. If $\left(\tau^{\mathrm{h}}, \mathbf{V}^{\prime}\right)$ is obtained from $\left(\pi^{\mathrm{f}}, \mathbf{V}\right)$ by any non empty set of operation, we can not reverse those changes and go back to $\left(\pi^{f}, \mathbf{V}\right)$ by using any sequence of these operations. This is because each of them increases the quantity $\max \{|\mathrm{B}|: \mathrm{B} \in \pi\} \cup\left\{\left|\mathrm{V}_{\mathbf{j}}\right|: \mathbf{j}=1,2, \ldots, r\right\}$, the maximum of the sizes of blocks of the partition together with those of the composition. The poset $\mathrm{Q}_{\mathrm{n}, \mathrm{r}}(\mathrm{G})$ has a least element $\widehat{0}=1|2| \ldots \mid \mathrm{n} \|(\emptyset, \emptyset, \ldots, \emptyset)$. The maximal elements are of the form $\emptyset \| \mathbf{V}, \mathbf{V}$ being a composition of $[n]$. 
Example 2 Consider the set $\mathrm{Q}_{11,2}(\mathrm{G})$, where $\mathrm{G}=\mathrm{F}_{5}^{\times}$. Its element

$$
25^{2}\left|34^{3}\right| 67^{4} 8^{3} 9 \mid 1011^{2} \|(\{1\}, \emptyset)
$$

comes from

$$
\pi^{f}|| \mathbf{V}=25^{2}\left|34^{3}\right| 67^{4}\left|89^{2}\right| 1011^{2} \|(\{1\}, \emptyset)
$$

by an operation of type $(1)$, because $67^{4} 8^{3} 9=\left(67^{2}\right)\left(89^{2}\right)^{3}$. Similarly,

$$
34^{3}\left|67^{4}\right| 89^{2} \|(\{1,2,5,10,11\}, \emptyset)
$$

is obtained from $\pi^{f} \| \mathbf{V}$ by an operation of type (2).

It is easy to check the following proposition, that gives an equivalente way of defining the partial order on $Q_{n, r}(G)$.

Proposition 1 Let $\left(\pi^{f}, \mathbf{V}\right)$ and $\left(\tau^{h}, \mathbf{V}^{\prime}\right)$ be two elements of $Q_{n, r}(G)$. We have that $\left(\pi^{f}, \mathbf{V}\right) \leq\left(\tau^{h}, \mathbf{V}^{\prime}\right)$ if and only if

1. For every $s=1,2, \ldots, r, V_{s} \subseteq V_{s}^{\prime}$.

2. Every block $B_{i}$ of $\pi=\left\{B_{1}, B_{2}, \ldots, B_{k}\right\}$ is either contained in $V_{s}^{\prime}$ for some $s \in[r]$ or in some block $B$ of $\tau$. In the second case, we have that $B=$ $\uplus_{B_{i} \subseteq B} B_{i}$, and for each $i$; $B_{i} \subseteq B$, there exists a $g_{i} \in G$ such that $\left.h\right|_{B_{i}}=$ $f_{B_{i}} * g_{i}$. In the case in which $\min B_{i}=\min B$, then $g_{i}=e$.

Lemma 1 Let $\left(\pi^{\mathrm{f}}, \mathrm{V}\right)$ be an element of $\mathrm{Q}_{\mathrm{n}, \mathrm{r}}(\mathrm{G}), \pi$ having exactly $\mathrm{k}$ blocks. Then the set of elements $\left(\tau^{\mathrm{h}}, \mathbf{V}^{\prime}\right)$ greater than or equal to $\left(\pi^{\mathrm{f}}, \mathbf{V}\right)$, such that $\tau$ has exactly $\mathrm{j} \leq \mathrm{k}$ blocks, is equal to the $\mathrm{r}$-Whitney number $\mathrm{W}_{\mathrm{m}, \mathrm{r}}(\mathrm{k}, \mathrm{j})$.

Proof. Order de blocks of $\pi$ according with their minimum elements, $\pi=$ $\left\{B_{1}, B_{2}, \ldots, B_{k}\right\}, \min _{i}<\min _{i+1}$. For each block $B$ of $\tau$ and $s=1,2, \ldots, r$, define the sets (subsets of $[k]$ ), $\widehat{B}:=\left\{i \mid B_{i} \subseteq B\right\}$ and $\widehat{V}_{s}^{\prime}=\left\{i \mid B_{i} \subseteq V_{s}^{\prime}\right\}$. By part (2) of Proposition 1, we have $h_{B}=\uplus_{i \in \widehat{B}} f_{B_{i}} * g_{i}$. We define the quotient coloration $h^{q}$ by $h_{\widehat{B}}^{q}(i)=g_{i}, i \in \widehat{B}$. Let $i_{0}$ be the minimum element of $\widehat{B}$. Since the elements of $\pi$ are listed according with their minimum element, the minimum of $B_{i_{0}}$ is also the minimum of the whole set $B$. Let $x=\min B_{i_{0}}$. Since $h_{B}$ and $f_{B_{i_{0}}}$ are both unital, we have

$$
e=h_{B}(x)=f_{B_{i_{0}}}(x) * g_{i_{0}}=e * g_{i_{0}}=g_{i_{0}}=h_{\widehat{B}}^{q}\left(i_{0}\right) .
$$

Then, $h_{\widehat{B}}^{q}$ is unital for every $\widehat{B} \in \widehat{\tau}$, and uniquely obtained from $h$ and $f$. By part (2) of Proposition 1, $\left(\widehat{\tau}^{\natural q}, \widehat{\mathbf{V}}^{\prime}\right)$ is a colored partition-composition pair on 
[k]. The correspondence $\left(\tau^{\mathrm{h}}, \mathbf{V}^{\prime}\right) \mapsto\left(\widehat{\tau}^{\mathrm{h}}, \widehat{\mathbf{V}}^{\prime}\right)$ is a bijection, and we have that $|\tau|=j=|\widehat{\tau}|$. The inverse $\left(\widehat{\tau}^{h^{q}}, \widehat{\mathbf{V}}^{\prime}\right) \mapsto\left(\tau^{h}, \mathbf{V}^{\prime}\right)$ is obtained by making

$$
\begin{aligned}
\mathrm{B} & :=\uplus_{i \in \widehat{B}} B_{i} \\
h_{B} & :=\uplus_{i \in \widehat{B}} f_{B_{i}} * h^{q}(i) \\
V_{s}^{\prime} & :=V_{s} \uplus\left(\uplus_{i \in \widehat{V}_{s}^{\prime}} B_{i}\right), s=1,2, \ldots, r .
\end{aligned}
$$

Example 3 As an example of the bijection in Lemma 1, let

$$
\pi^{f}\left\|\mathbf{V}=25^{2}\left|34^{3}\right| 67^{4}\left|89^{2}\right| 1011^{2}\right\|(\{1\}, \emptyset)
$$

and

$$
\tau^{\mathrm{h}}\left\|\mathbf{V}^{\prime}=67^{4} 8^{3} 910^{2} 11^{4}\right\|(\{1,2,5\},\{3,4\}) .
$$

We shall verify that $\pi^{\mathrm{f}}\left\|\mathbf{V} \leq \tau^{\mathrm{h}}\right\| \mathbf{V}^{\prime}$ and construct $\widehat{\tau}^{\mathrm{h}} \| \widehat{\mathbf{V}}^{\prime}$.

Enumerating the blocks of $\pi$ we have $B_{1}=\{2,5\}, B_{2}=\{3,4\}, B_{3}=\{6,7\}$, $\mathrm{B}_{4}=\{8,9\}, \mathrm{B}_{5}=\{10,11\}$. Since

$$
67^{4} 8^{3} 910^{2} 11^{4}=\left(67^{4}\right)^{1}\left(89^{2}\right)^{3}\left(1011^{2}\right)^{2}
$$

and

$$
(\{1,2,5\},\{3,4\})=(\{1\} \cup\{2,5\}, \emptyset \cup\{3,4\})=\left(\{1\} \cup B_{1}, \emptyset \cup B_{2}\right),
$$

we have that $\pi^{\mathrm{f}}\left\|\mathbf{V} \leq \tau^{\mathrm{h}}\right\| \mathbf{V}^{\prime}$, and get $\widehat{\tau}^{\mathrm{h}}\left\|\widehat{\mathbf{V}}^{\prime}=3^{1} 4^{3} 5^{2}\right\|(\{1\},\{2\})$.

The poset $Q_{n, r}(G)$ is ranked. The rank of an element is $n-k, k$ being the number of blocks of the colored partition.

Theorem 2 The sum of the Möbius function over the elements of rank $n-k$ in $\mathrm{Q}_{\mathrm{n}, \mathrm{r}}(\mathrm{G})$ gives us the $\mathrm{r}$-Withney number of the first kind $w_{\mathrm{m}, \mathrm{r}}(\mathrm{n}, \mathrm{k})$. Denoting by $\mu$ the Möbius function of the poset $Q_{n, r}(G)$, we have

$$
w_{\mathrm{m}, \mathrm{r}}(\mathrm{n}, \mathrm{k})=\sum_{\left(\pi^{\mathrm{f}}, \mathbf{V}\right),|\pi|=k} \mu\left(\widehat{0},\left(\pi^{\mathrm{f}}, \mathbf{V}\right)\right)
$$

Proof. Define the matrix $\mathrm{C}(n, k)$ to be the right hand side of Eq. (7). It is enough to prove that

$$
\sum_{0 \leq k \leq n} C(n, k) W_{m, r}(k, j)=\delta_{n, j}
$$


Let $\left(\tau^{h}, V^{\prime}\right)$ be an element of $Q_{n, r}(G, j)$. By properties of the Möbius function we have that

$$
\sum_{\widehat{0} \leq\left(\pi^{\mathrm{f}}, \mathbf{V}\right) \leq\left(\tau^{\mathrm{h}}, \mathbf{V}^{\prime}\right)} \mu\left(\widehat{0},\left(\pi^{\mathrm{f}}, \mathbf{V}\right)\right)=\delta\left(\widehat{0},\left(\tau^{\mathrm{h}}, \mathbf{V}^{\prime}\right)\right)=\delta_{\mathrm{n}, \mathrm{j}} .
$$

Summing over all the elements of $Q_{n, r}(G, j)$, interchanging sums and classifying by the size of $\pi$, we get

$$
\begin{aligned}
& \delta_{n, j}=\sum_{\left(\tau^{\mathrm{h}}, \mathbf{V}^{\prime}\right) \in Q_{n, r}(G, j)} \sum_{\hat{0} \leq\left(\pi^{\mathrm{f}}, \mathbf{V}\right) \leq\left(\tau^{\mathrm{h}}, \mathbf{V}^{\prime}\right)} \mu\left(\widehat{0},\left(\pi^{\mathrm{f}}, \mathbf{V}\right)\right) \\
& =\sum_{\mathrm{k}=0}^{n} \sum_{\left(\pi^{\mathrm{f}}, \mathbf{V}\right) \in \mathrm{Q}_{\mathrm{n}, \mathrm{r}}(\mathrm{G}, \mathrm{k})}\left(\sum_{\substack{\left(\tau^{\mathrm{h}} \mathrm{V} \mathbf{V}^{\prime}\right) \geq\left(\pi^{\mathrm{f}}, \mathbf{V}\right) \\
\left(\tau^{\mathrm{h}}, \mathbf{V}^{\prime}\right) \in \mathrm{Q}_{n}, \mathrm{r}(\mathrm{G}, \mathfrak{j})}} \mu\left(\widehat{0},\left(\pi^{\mathrm{f}}, \mathbf{V}\right)\right)\right) \\
& =\sum_{\mathrm{k}=0}^{n} \sum_{\left(\pi^{\mathrm{f}}, \mathbf{V}\right) \in \mathrm{Q}_{\mathrm{n}, \mathrm{r}}(\mathrm{G}, \mathrm{k})} \mu\left(\widehat{0},\left(\pi^{\mathrm{f}}, \mathbf{V}\right)\right)\left(\sum_{\substack{\left(\tau^{\mathrm{h}}, \mathbf{V}^{\prime}\right) \geq\left(\pi^{\mathrm{f}}, \mathbf{V}\right) \\
\left(\tau^{\mathrm{h}}, \mathbf{V}^{\prime}\right) \in \mathrm{Q}_{n}, \mathrm{r}(\mathrm{G}, \mathbf{j})}} 1\right) \\
& =\sum_{k=0}^{n} \sum_{\left(\pi^{f}, \mathbf{V}\right) \in Q_{n}, r(G, k)} \mu\left(\widehat{0},\left(\pi^{f}, \mathbf{V}\right)\right)\left|\left\{\left(\tau^{h}, \mathbf{V}^{\prime}\right)\left|\left(\tau^{h}, \mathbf{V}^{\prime}\right) \geq\left(\pi^{f}, \mathbf{V}\right),\right| \tau \mid=j\right\}\right| \\
& =\sum_{k=0}^{n} C(n, k) W_{m, r}(k, j) \text {. }
\end{aligned}
$$

The last equality obtained by Lemma 1 .

Example 4 The poset of colored partition-set pairs, $\mathrm{Q}_{\mathrm{n}, 1}(\mathrm{G})$, is isomorphic to the classical Dowling lattice. See Figure 8 for the Hasse diagram of the poset $\mathrm{Q}_{2,2}(\mathrm{G}), \mathrm{G}$ being the group with two elements $\mathrm{G}=\{-1,1\}$ (writing $\overline{2}$ instead of $\left.2^{-1}\right)$. From the diagram we get its Möbius function and obtain $w_{2,2}(2,2)=$ $1, w_{2,2}(2,1)=-6$, and $w_{2,2}(2,0)=8 . \mathrm{Q}_{n, r}(\{-1,1\})$ generalizes the signed partitions poset, $\bar{\Pi}[\mathrm{n}]$ obtained for $\mathrm{r}=1, \bar{\Pi}[\mathrm{n}]=\mathrm{Q}_{\mathrm{n}, 1}(\{-1,1\})$.

\section{The r-Dowling polynomials}

Cheon and Jung [8] defined the $r$-Dowling polynomials of degree $\boldsymbol{n}$ by

$$
\mathcal{D}_{\mathrm{m}, \mathrm{r}}(\mathrm{n}, \mathrm{u}):=\sum_{\mathrm{k}=0}^{\mathrm{n}} \mathrm{W}_{\mathrm{m}, \mathrm{r}}(\mathrm{n}, \mathrm{k}) \mathrm{u}^{\mathrm{k}} .
$$




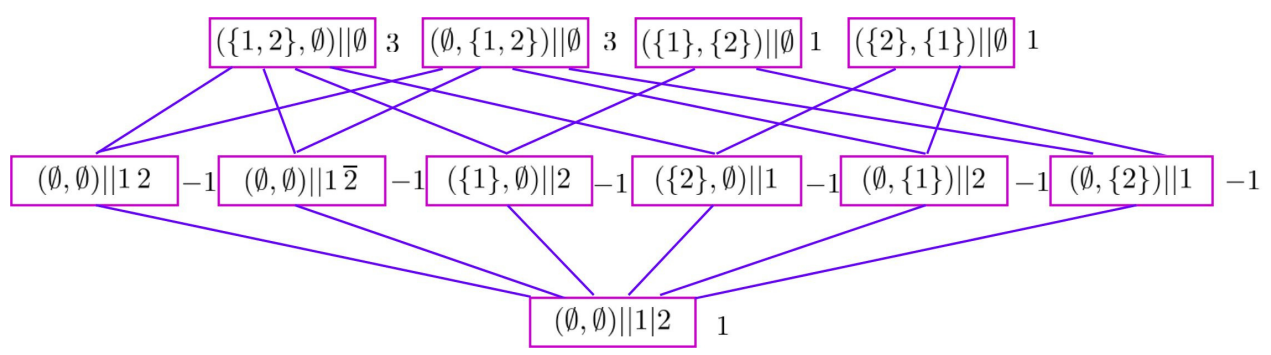

Figure 8: Poset $Q_{2,2}(G), G=\{-1,1\}$.

They found some combinatorial identities by means of Riordan arrays. Let us define the following generating function

$\mathcal{H}_{\mathrm{m}, \mathrm{r}}(\mathrm{t})=\mathcal{H}(\mathrm{t} ; x, y)=e^{\mathrm{t} \mathcal{D}} y x^{r}=\sum_{n=0}^{\infty} \frac{t^{n}}{n !} \mathcal{D}^{n} y x^{r}=\sum_{n=0}^{\infty} \frac{t^{n}}{n !} \sum_{k=0}^{n} W_{m, r}(n, k) y x^{k m+r}$.

It is easy to show the following

Lemma 2 We have the identities

$$
\begin{aligned}
\mathcal{D}^{n} y x^{r} & =y x^{r} \mathcal{D}_{m, r}\left(n, x^{m}\right), \\
\mathcal{H}_{m, r}(t) & =e^{t} \mathcal{D} y x^{r}=y x^{r} \sum_{n=0}^{\infty} \frac{t^{n}}{n !} \mathcal{D}_{m, r}\left(n, x^{m}\right) .
\end{aligned}
$$

Theorem 3 The exponential generating function for the r-Dowling polynomials is

$$
\sum_{n=0}^{\infty} \mathcal{D}_{m, r}(n, u) \frac{t^{n}}{n !}=\exp \left(r t+u \frac{e^{m t}-1}{m}\right) .
$$

Proof. Since $\mathcal{D}$ is a derivation, it is easy to show that the operator $e^{\mathrm{t} \mathcal{D}}$ is multiplicative. Hence

$$
\begin{aligned}
\mathcal{H}_{m, r}^{\prime}(t)=e^{t \mathcal{D}} \mathcal{D} y x^{r} & =e^{t \mathcal{D}}\left(y x^{m+r}+r y x^{r}\right)=\left(e^{\mathfrak{t} \mathcal{D}} y x^{r}\right) \cdot\left(e^{t \mathcal{D}} x\right)^{m}+r e^{t \mathcal{D}} y x^{r} \\
& =\mathcal{H}_{m, r}(t)\left(x^{m} e^{\mathrm{mt}}+r\right) .
\end{aligned}
$$

From that,

$$
\frac{\mathcal{H}_{\mathrm{m}, \mathrm{r}}^{\prime}(\mathrm{t})}{\mathcal{H}_{\mathrm{m}, \mathrm{r}}(\mathrm{t})}=\frac{\mathrm{d}}{\mathrm{dt}} \ln \left(\mathcal{H}_{\mathrm{m}, \mathrm{r}}(\mathrm{t})\right)=x^{\mathrm{m}} e^{\mathrm{mt}}+\mathrm{r}
$$


Integrating and using the initial condition $\mathcal{H}_{\mathrm{m}, \mathrm{r}}(0)=y x^{\mathrm{r}}$, we get:

$$
\mathcal{H}_{\mathrm{m}, \mathrm{r}}(\mathrm{t})=y x^{\mathrm{r}} \exp \left\{\mathrm{rt}+\frac{x^{\mathrm{m}}}{\mathrm{m}}\left(e^{\mathrm{mt}}-1\right)\right\} .
$$

By Lemma 2, making $u=x^{m}$ we get the result.

Theorem 4 The $r$-Dowling polynomials satisfy the following relation for any integers $r, \ell \geq 0$

$$
\mathcal{D}_{\mathfrak{m}, \mathrm{r}+\ell}(\mathrm{n}, \mathrm{u})=\sum_{k=0}^{n}\left(\begin{array}{l}
n \\
k
\end{array}\right) \ell^{n-k} \mathcal{D}_{\mathfrak{m}, \mathrm{r}}(k, u) .
$$

Proof. Let $\widehat{\mathcal{D}}_{m, r}(n, x, y):=\sum_{k=0}^{n} W_{m, r}(n, k) y x^{k m+r}$. Then

$$
\begin{aligned}
\mathcal{H}_{\mathrm{m}, \mathrm{r}+\ell}(\mathrm{t}) & =e^{\mathrm{t} \mathcal{D}} y x^{\mathrm{r}+\ell}=\left(e^{\mathrm{t} \mathcal{D}} y x^{r}\right)\left(e^{\mathrm{t} \mathcal{D}} x^{\ell}\right) \\
& =x^{\ell} e^{\ell t} \mathcal{H}_{\mathrm{m}, \mathrm{r}}(\mathrm{t})=x^{\ell} \sum_{n=0}^{\infty} \frac{t^{n}}{n !}\left(\sum_{j=0}^{n}\left(\begin{array}{l}
n \\
j
\end{array}\right) \ell^{n-j} \widehat{\mathcal{D}}_{\mathrm{m}, \mathrm{r}}(j, x, y)\right) .
\end{aligned}
$$

Therefore

$$
\widehat{\mathcal{D}}_{m, r}(n, x, y)=x^{\ell} \sum_{j=0}^{n}\left(\begin{array}{l}
n \\
j
\end{array}\right) \ell^{n-j} \widehat{\mathcal{D}}_{m, r}(j, x, y) .
$$

Modifying a bit this equality we obtain the desired result.

In particular, if $\ell=1$ then

$$
\mathcal{D}_{\mathfrak{m}, \mathrm{r}+1}(\mathrm{n}, \mathrm{u})=\sum_{\mathrm{k}=0}^{\mathrm{n}}\left(\begin{array}{l}
\mathrm{n} \\
k
\end{array}\right) \mathcal{D}_{\mathrm{m}, \mathrm{r}}(\mathrm{k}, \mathrm{u}) .
$$

In Theorem 5 we generalize the beautiful relation given by Spivey [34] for the Bell numbers $B_{n}$. The Spivey's formula says that:

$$
B_{n+m}=\sum_{k=0}^{n} \sum_{j=0}^{m} j^{n-k} S(m, j)\left(\begin{array}{l}
n \\
k
\end{array}\right) B_{k},
$$

where $S(n, j)$ is the Stirling number of the second kind. We generalize this identity for the $r$-Whitney numbers by using differential operators. 
Theorem 5 The r-Dowling polynomials satisfy the following formula

$$
\mathcal{D}_{m, r}(n+h, u)=\sum_{k=0}^{n} \sum_{j=0}^{h}\left(\begin{array}{l}
n \\
k
\end{array}\right) \mathcal{D}_{m, r}(k, u) W_{m, r}(h, j) u^{j} j^{n-k} m^{n-k} .
$$

Proof. We compute the derivative in two ways. First, in a direct way

$$
\frac{\mathrm{d}^{\mathrm{h}}}{\mathrm{d} \mathrm{t}^{\mathrm{h}}} \mathcal{H}_{\mathrm{m}, \mathrm{r}}(\mathrm{t})=y x^{\mathrm{r}} \sum_{\mathrm{n}=0}^{\infty} \mathcal{D}_{\mathrm{m}, \mathrm{r}}\left(\mathrm{n}+\mathrm{h}, \mathrm{x}^{\mathrm{m}}\right) \frac{\mathrm{t}^{\mathrm{n}}}{\mathrm{n} !},
$$

and secondly, by using the identity $\frac{\mathrm{d}^{\mathrm{h}}}{\mathrm{dt}^{\mathrm{h}}} \mathrm{e}^{\mathrm{t} \mathcal{D}}=e^{\mathrm{t} \mathcal{D}} \mathcal{D}^{\mathrm{h}}$ and Lemma 2

$$
\begin{aligned}
\frac{\mathrm{d}^{\mathrm{h}}}{\mathrm{d} \mathrm{t}^{\mathrm{h}}} \mathcal{H}_{\mathrm{m}, \mathrm{r}}(\mathrm{t}) & =\frac{\mathrm{d}^{\mathrm{h}}}{\mathrm{d} \mathrm{t}^{\mathrm{h}}} e^{\mathrm{t} \mathcal{D}} y x^{\mathrm{r}}=e^{\mathrm{t} \mathcal{D}} \mathcal{D}^{\mathrm{h}} y x^{\mathrm{r}}=e^{\mathrm{t} \mathcal{D}} y x^{\mathrm{r}} \mathcal{D}_{\mathrm{m}, \mathrm{r}}\left(\mathrm{h}, x^{\mathrm{m}}\right) \\
& =y x^{\mathrm{r}}\left(\sum_{n=0}^{\infty} \mathcal{D}_{\mathrm{m}, \mathrm{r}}\left(\mathrm{n}, x^{\mathrm{m}}\right) \frac{\mathrm{t}^{\mathrm{n}}}{\mathrm{n} !}\right)\left(\mathcal{D}_{\mathrm{m}, \mathrm{r}}\left(\mathrm{h}, x^{\mathrm{m}} e^{\mathrm{mt}}\right)\right) .
\end{aligned}
$$

Making the change $u=x^{m}$ and from the generating functions in Equations (4) and (10), we obtain

$$
\sum_{n=0}^{\infty} \mathcal{D}_{m, r}(n+h, u) \frac{t^{n}}{n !}=\left(\sum_{n=0}^{\infty} \mathcal{D}_{m, r}(n, u) \frac{t^{n}}{n !}\right)\left(\mathcal{D}_{m, r}\left(h, u e^{m t}\right)\right)
$$

Expanding $\mathcal{D}_{m, r}\left(h, u e^{m t}\right)$,

$$
\begin{aligned}
\mathcal{D}_{m, r}\left(h, u e^{m t}\right) & =\sum_{j=0}^{h} W_{m, r}(h, j) u^{j} e^{m j t} \\
& =\sum_{j=0}^{h} W_{m, r}(h, j) u^{j} \sum_{k=0}^{\infty} m^{k} j^{k} \frac{t^{k}}{k !}=\sum_{k=0}^{\infty}\left(\sum_{j=0}^{h} W_{m, r}(h, j) m^{k} u^{j} j^{k}\right) \frac{t^{k}}{k !} .
\end{aligned}
$$

By plugging it into Equation (11), computing the Cauchy product and equating coefficients, we obtain the result.

The above identity was proved by using a different approach in [37]. Moreover, this identity is a particular case of the main result of [36].

From Theorem 5 we obtain the following convolution formula.

Corollary 1 For $0 \leq \mathrm{s} \leq \mathrm{n}+\mathrm{h}$, we have

$$
W_{m, r}(n+h, s)=\sum_{k=0}^{n} \sum_{j=0}^{h}\left(\begin{array}{l}
n \\
k
\end{array}\right) W_{m, r}(h, j) W_{m, r}(k, s-j)(j m)^{n-k} .
$$


In particular, if $r=1$ we obtain the Theorem 4.3 of [13]. By setting $h=1$ in Theorem 5 and Corollary 1 we obtain the Corollary 2.

Corollary 2 The $\mathrm{r}$-Dowling polynomials satisfy the following recursive formula

$$
\mathcal{D}_{m, r}(n+1, u)=r \mathcal{D}_{m, r}(n, u)+u \sum_{j=0}^{n}\left(\begin{array}{l}
n \\
j
\end{array}\right) m^{n-j} \mathcal{D}_{m, r}(j, u) .
$$

Therefore, the $\mathrm{r}$-Whitney numbers of the second kind satisfy the recursive formula

$$
W_{m, r}(n+1, k)=r W_{m, r}(n, k)+\sum_{j=k-1}^{n}\left(\begin{array}{l}
n \\
j
\end{array}\right) m^{n-j} W_{m, r}(j, k-1) .
$$

The above corollary was proved in [8] by using Riordan arrays.

Theorem 6 The r-Dowling polynomials satisfy the following formula

$$
\mathcal{D}_{m, r}(n, u)=\sum_{j=0}^{n}\left(\begin{array}{l}
n \\
j
\end{array}\right)(r-s)^{n-j} D_{m, s}(j, u) .
$$

Proof. From Lemma 2 we have

$$
\mathcal{H}_{\mathrm{m}, \mathrm{r}}(\mathrm{t})=e^{\mathrm{t} \mathcal{D}} y x^{\mathrm{r}}=e^{\mathrm{t} \mathcal{D}} y x^{\mathrm{s}} e^{\mathrm{t} \mathcal{D}} x^{\mathrm{r}-\mathrm{s}}=x^{\mathrm{r}-\mathrm{s}} e^{(\mathrm{r}-\mathrm{s}) \mathrm{t}} \mathcal{H}_{\mathrm{m}, \mathrm{s}}(\mathrm{t}) .
$$

By the Cauchy product we obtain

$$
\widehat{\mathcal{D}}_{m, r}(n, x, y)=x^{r-s} \sum_{j=0}^{n}\left(\begin{array}{l}
n \\
j
\end{array}\right)(r-s)^{n-j} \widehat{\mathcal{D}}_{m, s}(j, x, y) .
$$

Therefore, we get the desired result.

In particular if $s=1$ we have (see Theorem 5.2 of [8])

$$
\mathcal{D}_{m, r}(n, x)=\sum_{j=0}^{n}(r-1)^{n-j}\left(\begin{array}{l}
n \\
j
\end{array}\right) D_{m}(j, x),
$$

where $D_{m}(n, u)$ are the Dowling polynomials, i.e.,

$$
D_{m}(n, u)=\sum_{k=0}^{n} W_{m}(n, k) u^{k}
$$


Corollary 3 The r-Whitney numbers satisfy the following formula

$$
W_{m, r}(n, k)=\sum_{j=0}^{n}\left(\begin{array}{l}
n \\
j
\end{array}\right)(r-s)^{n-j} W_{m, s}(j, k) .
$$

In particular, if $\mathrm{s}=1$ we have

$$
W_{m, r}(n, k)=\sum_{j=0}^{n}\left(\begin{array}{l}
n \\
j
\end{array}\right)(r-1)^{n-j} W_{m}(j, k) .
$$

Proof. From Theorem 6

$$
\begin{aligned}
\sum_{k=0}^{n} W_{m, r}(n, k) y x^{m k+r} & =x^{r-s} \sum_{j=0}^{r}\left(\begin{array}{l}
n \\
j
\end{array}\right)(r-s)^{n-j} \sum_{k=0}^{j} W_{m, s}(j, k) y x^{k m+s} \\
& =\sum_{k=0}^{n} \sum_{j=0}^{n}\left(\begin{array}{l}
n \\
j
\end{array}\right)(r-s)^{n-j} W_{m, s}(j, k) y x^{k m+r} .
\end{aligned}
$$

By equating coefficients, we obtain the result.

\section{The Sheffer family of [m]-Touchard polynomials and r-Dowling polynomials}

From the generating function of the r-Dowling polynomials (Theorem 3), making $r=0$, we get the generating function of the [m]-Touchard polynomials

$$
\sum_{n=0}^{\infty} T_{n}^{[m]}(x) \frac{t^{n}}{n !}=\exp \left(x \frac{e^{m t}-1}{m}\right) .
$$

From this is immediate that they are of binomial type ([32]). From Equation (9) we have the following identity relating them with the classical Dowling polynomials

$$
\mathcal{D}_{\mathfrak{m}, 1}(n, x)=\mathcal{D}_{\mathfrak{m}}(n, x)=\sum_{k=0}^{n}\left(\begin{array}{l}
n \\
k
\end{array}\right) T_{k}^{[m]}(x) .
$$

and hence,

$$
\mathrm{T}_{\mathrm{n}}^{[\mathrm{m}]}(x)=\sum_{k=0}^{n}\left(\begin{array}{l}
n \\
k
\end{array}\right)(-1)^{n-k} \mathcal{D}_{\mathfrak{m}}(k, x) .
$$


Our goal is to find the umbral inverse of [m]-Touchard sequence, i.e., the polynomial family $\widehat{T}_{n}^{[m]}(x)=\sum_{k=1}^{n} c_{n, k} x^{k}$ satisfying

$$
\begin{aligned}
& \mathrm{T}_{\mathrm{n}}^{[\mathrm{m}]}\left(\widehat{\mathbf{T}}^{[\mathrm{m}]}(\mathrm{x})\right):=\sum_{k=1}^{n} S^{[\mathrm{m}]}(\mathrm{n}, \mathrm{k}) \widehat{\mathrm{T}}_{\mathrm{k}}^{[\mathrm{m}]}(\mathrm{x})=x^{\mathrm{n}}, \forall \mathrm{n}=0,1,2 \ldots \\
& \widehat{\mathrm{T}}_{\mathrm{n}}^{[\mathrm{m}]}\left(\mathbf{T}^{[\mathrm{m}]}(\mathrm{x})\right):=\sum_{\mathrm{k}=1}^{n} c_{n, k} \mathrm{~T}_{k}^{[\mathrm{m}]}(\mathrm{x})=x^{\mathrm{n}}, \forall \mathrm{n}=0,1,2 \ldots
\end{aligned}
$$

Let $\mathcal{O}(t):=\left(e^{m t}-1\right) / m$, and consider its compositional inverse

$$
\overline{\mathcal{O}}(t)=\ln (1+m x)^{\frac{1}{m}} .
$$

Let $\partial_{x}$ be the derivative operator acting on the polynomial ring $\mathbb{C}[x]$. Let $\mathcal{O}\left(\partial_{x}\right)$ and $\overline{\mathcal{O}}\left(\partial_{x}\right)$ be the shift-invariant operators acting also on the polynomial ring $\mathbb{C}[x]$,

$\mathcal{O}\left(\partial_{x}\right)=\frac{e^{m \partial_{x}}-I}{m}=\frac{1}{m} \sum_{k=1}^{\infty} m^{k} \frac{\partial_{x}^{k}}{k !}=\frac{E^{m}-I}{m}$,

$\overline{\mathcal{O}}\left(\partial_{x}\right)=\ln \left(1+m \partial_{x}\right)^{\frac{1}{m}}=\frac{1}{m} \sum_{k=1}^{\infty}(-1)^{k} m^{k}(k-1) ! \frac{\partial_{x}^{k}}{k !}=\sum_{k=1}^{\infty}(-1)^{k} m^{k-1}(k-1) ! \frac{\partial_{x}^{k}}{k !}$.

Here we denote by $E^{a}$ the shift operator $E^{a} p(x)=p(x+a)$. By the classical theory of umbral calculus, $\widehat{T}_{n}^{[m]}(x)$ and $T_{n}^{[m]}(x)$ are the sequences associated to the operators $\frac{E^{m}-I}{m}$, and $\ln \left(1+m \partial_{x}\right)^{\frac{1}{m}}$, respectively. So

$$
\begin{aligned}
\frac{E^{m}-I}{m} \widehat{T}_{n}^{[m]}(x)=\frac{\widehat{T}_{n}^{[m]}(x+m)-\widehat{T}_{n}^{[m]}(x)}{m} & =n \widehat{T}_{n-1}^{[m]}(x) \\
\ln \left(1+m \partial_{x}\right)^{\frac{1}{m}} T_{n}^{[m]}(x) & =n T_{n-1}^{[m]}(x) .
\end{aligned}
$$

The derivatives of the formal power series $\mathcal{O}(x)$ and $\overline{\mathcal{O}}(x)$ are $\mathcal{O}^{\prime}(x)=e^{\mathrm{m} x}$ and $(\overline{\mathcal{O}}(x))^{\prime}=\frac{1}{1+m x}$, respectively. The recurrence formula for families of binomial type ([32] Corollary 1 of Theorem 8), gives us

$$
\begin{aligned}
\widehat{T}_{n}^{[m]}(x) & =x E^{-m} \widehat{T}_{n-1}^{[m]}(x)=x \widehat{T}_{n-1}^{[m]}(x-m), \\
T_{n}^{[m]}(x) & =x\left(1+m \partial_{x}\right) T_{n-1}^{[m]}(x)=x T_{n-1}^{[m]}(x)+m x \partial_{x} T_{n-1}^{[m]}(x) .
\end{aligned}
$$


From Equation (16) we obtain the polynomial family

$$
\widehat{T}_{n}^{[m]}(x)=x(x-m)(x-2 m) \cdots(x-(n-1) m) .
$$

From Equation (16) we also have the recurrence for [m]-Stirling numbers of the second kind

$$
S^{[m]}(n, k)=S^{[m]}(n-1, k-1)+k m S^{[m]}(n-1, k) .
$$

We define the $[\mathrm{m}]$-Stirling numbers of the first kind as the coefficients connecting $\widehat{T}_{n}^{[m]}(x)$ with the powers.

Definition 3 We define the $[\mathrm{m}]$-Stirling numbers of the first kind $\mathrm{s}^{[\mathrm{m}]}(\mathrm{n}, \mathrm{k})$ as the coefficients in the expansion of $\widehat{T}_{n}^{[m]}(x)=x(x-m)(x-2 m) \cdots(x-(n-1) m)$ in terms of the power sequence, that is,

$$
x(x-m)(x-2 m) \cdots(x-(n-1) m)=\sum_{k=1}^{n} s^{[m]}(n, k) x^{k} .
$$

Remark 2 The [m]-Stirling numbers of the first kind have the following combinatorial interpretation in terms of the Möbius function. Considering $\mathrm{Q}_{\mathrm{n}, \mathrm{o}}(\mathrm{G})$, the subposet of $Q_{n, r}$ of elements of the form $\left(\pi^{f},(\emptyset, \emptyset, \ldots, \emptyset)\right)$, $s^{[m]}(n, k)$ is the sum of its Möbius function over the elements of rank $\mathrm{n}-\mathrm{k}$. The proof of this fact is completely analogous of that of Theorem 2.

Remark 3 From the generating function in Theorem 3,

$$
\sum_{n=0}^{\infty} \mathcal{D}_{m, r}(n, x) \frac{t^{n}}{n !}=e^{r x} \exp \left(x \frac{e^{m t}-1}{m}\right),
$$

we have that the $\mathrm{r}$-Dowling polynomials, $\mathcal{D}_{\mathfrak{n}}^{[\mathrm{m}, \mathrm{r}]}(\mathrm{x}):=\mathcal{D}_{\mathrm{m}, \mathrm{r}}(\mathrm{n}, \mathrm{x})$ are a Sheffer family relative to the $[\mathrm{m}]-$ Touchard, associated to the pair of generating functions $\left(\frac{1}{(1+m x)^{r / m}}, \ln (1+m x)^{1 / m}\right)$,

$$
\left\langle\frac{1}{(1+m x)^{r / m}}, \ln (1+m x)^{1 / m}\right\rangle=\left\langle e^{r x}, \frac{e^{m x}-1}{m}\right\rangle^{-1},
$$

being the inverse of $\left\langle\mathrm{e}^{\mathrm{rx}}, \frac{\mathrm{e}^{\mathrm{mx}}-1}{\mathrm{~m}}\right\rangle$ as an exponential Riordan array, see Equation (23) bellow. For that, see [32], and [31] Theorem 2.3.4. Hence, we have the binomial identity (see [31], Theorem 2.3.9)

$$
\mathcal{D}_{n}^{[m, r]}(x+y)=\sum_{k=0}^{n}\left(\begin{array}{l}
n \\
k
\end{array}\right) \mathcal{D}_{k}^{[m, r]}(x) T_{n-k}^{[m]}(y) .
$$


Its umbral inverse $\widehat{\mathcal{D}}_{n}^{[m, r]}(x)$ is Sheffer relative to $\widehat{T}_{n}^{[m]}(x)$. It is associated to the pair $\left(e^{r x}, \frac{e^{m x}-1}{m}\right)$.

From that we get next theorem

Theorem 7 The umbral inverse of the $\mathrm{r}$-Dowling Sheffer sequence is equal to

$$
\widehat{\mathcal{D}}_{n}^{[m, r]}(x)=E^{-r} \widehat{T}_{n}^{[m]}(x)=(x-r)(x-r-m) \cdots(x-r-(k-1) m) .
$$

The r-Dowling sequence satisfies the identity

$$
\mathcal{D}_{n}^{[m, r]}(x)=\sum_{k=0}^{n} \frac{r(r-m)(r-2 m) \cdots(r-(k-1) m)}{k !} \partial_{x}^{k} T_{n}^{[m]}(x) .
$$

Proof. Since $\widehat{\mathcal{D}}_{n}^{[m, r]}(x)$ is associated to $\left(e^{r x}, \frac{e^{m x}-1}{m}\right)$, it is equal to the inverse of the operator $e^{r \partial_{x}}=E^{r}$ applied to $\widehat{T}_{n}^{[m]}(x)$ (see [31], Theorem 2.3.6). For the same reason we have that $\mathcal{D}_{n}^{[m, r]}(x)=\left(1+m \partial_{x}\right)^{r / m} T_{n}^{[m]}(x)$. Expanding the operator $\left(1+m \partial_{x}\right)^{r / m}$ by the binomial formula we obtain the result.

\section{Some applications from Riordan arrays}

The $r$-Whitney numbers can be defined using exponential Riordan arrays. An infinite lower triangular matrix is called a Riordan array [33] if its kth column satisfies the generating function $g(z)(f(z))^{k}$ for $k \geq 0$, where $g(z)$ and $f(z)$ are formal power series with $g(0) \neq 0, f(0)=0$ and $f^{\prime}(0) \neq 0$. The matrix corresponding to the pair $f(z), g(z)$ is denoted by $(g(z), f(z))$. The product of two Riordan arrays $(g(z), f(z))$ and $(h(z), l(z))$ is defined by

$$
(g(z), f(z)) *(h(z), l(z))=(g(z) h(f(z)), l(f(z))) .
$$

The set of the Riordan matrices is a group under the operator "* " [33]. The identity element is $I=(1, z)$, and the inverse of $(g(z), f(z))$ is

$$
(g(z), f(z))^{-1}=(1 /(g \circ \bar{f})(z), \bar{f}(z)),
$$

Sometimes, it is useful to use exponential generating functions instead of ordinary generating functions when we apply a Riordan array method. We call the resulting array an exponential Riordan array and we denote it by $\langle g(z), f(z)\rangle$. Its column $k$ has generating function $g(z)(f(z))^{k} / k !, k=0,1,2, \ldots$ (cf. [35]). 
The r-Whitney numbers of the second kind are given by the exponential Riordan array:

$$
W_{2}:=\left[W_{m, r}(n, k)\right]_{n, k \geq 0}=\left\langle e^{r x}, \frac{e^{m x}-1}{m}\right\rangle
$$

Therefore, as we have already seen in Remark 3, the r-Dowling polynomial $\mathcal{D}_{n}^{[m, r]}(x)$ is a Sheffer sequence for

$$
\left((1+m x)^{-r / m}, \ln (1+m x)^{1 / m}\right) .
$$

In this section we give some explicit relations between the r-Dowling polynomials and the Bernoulli and Euler polynomials by using the connection constants $a_{n, k}$ in the expression $r_{n}(x)=\sum_{k=0}^{n} a_{n, k} s_{k}(x)$, where the polynomials $r_{n}(x)$ and $s_{n}(x)$ are Sheffer sequences. These constants can be determined by the umbral method [31, pp. 131] or equivalent by Riordan arrays [35, Theorem 6.4]. In particular, let $s_{n}(x)$ and $r_{n}(x)$ be Sheffer for $(g(t), f(t))$ and $(h(t), l(t))$, respectively. If $r_{n}(x)=\sum_{k=0}^{n} a_{n, k} s_{k}(x)$, then $a_{n, k}$ is the entry $(n, k)$-th of the Riordan array

$$
\left(\frac{g(\bar{l}(t))}{h(\bar{l}(t))}, f(\bar{l}(t))\right)
$$

The Bernoulli polynomials, $\mathcal{B}_{\mathfrak{n}}(x)$, are defined by the exponential generating function

$$
\sum_{n=0}^{\infty} \mathcal{B}_{n}(x) \frac{t^{n}}{n !}=\frac{t e^{x t}}{e^{t}-1} .
$$

The Bernoulli numbers, $\mathcal{B}_{\mathfrak{n}}$, are define by $\mathcal{B}_{\mathfrak{n}}:=\mathcal{B}_{\mathfrak{n}}(0)$. Moreover, the polynomials $\mathcal{B}_{n}(x)$ are Sheffer for $\left(\frac{e^{t}-1}{t}, t\right)$ (cf. $[31,14]$ ). The $r$-Whitney numbers of the first kind are defined by exponential generating function [24]:

$$
\sum_{n=k}^{\infty} w_{m, r}(n, k) \frac{z^{n}}{n !}=(1+m z)^{-\frac{r}{m}} \frac{\ln ^{k}(1+m z)}{m^{k} k !} .
$$

Theorem 8 For $\mathrm{n} \geq 0$ we have

$$
\mathcal{B}_{n}(x)=\sum_{k=0}^{n} \sum_{\ell=k}^{n}\left(\begin{array}{l}
n \\
\ell
\end{array}\right) \mathcal{B}_{n-\ell} w_{m, r}(\ell, k) \mathcal{D}_{k}^{[m, r]}(x) .
$$


Proof. If $\mathcal{B}_{\mathfrak{n}}(x)=\sum_{k=0}^{n} a_{n, k} \mathcal{D}_{k}^{[m, r]}(x)$, then from (24) and (25) we get

$$
\begin{aligned}
a_{n, k} & =\frac{1}{k !}\left[t^{n}\right]\left((1+m t)^{-r / m} \frac{t}{e^{t}-1} \cdot\left(\ln (1+m t)^{1 / m}\right)^{k}\right) \\
& =\left[t^{n}\right]\left((1+m t)^{-r / m} \frac{\ln ^{k}(1+m t)}{m^{k} k !} \frac{t}{e^{t}-1}\right) \\
& =\left[t^{n}\right]\left(\left(\sum_{n=k}^{\infty} w_{m, r}(n, k) \frac{t^{n}}{n !}\right)\left(\sum_{n=0}^{\infty} \mathcal{B}_{n}(x) \frac{t^{n}}{n !}\right)\right) \\
& =\left[t^{n}\right]\left(\sum_{n=0}^{\infty} \sum_{\ell=0}^{n}\left(\begin{array}{c}
n \\
\ell
\end{array}\right) \mathcal{B}_{n-\ell} w_{m, r}(\ell, k)\right) \frac{z^{n}}{n !} .
\end{aligned}
$$

Therefore, it is clear (26).

The Euler polynomials, $\mathcal{E}_{\mathfrak{n}}(x)$, are defined by the exponential generating function

$$
\sum_{n=0}^{\infty} \mathcal{E}_{n}(x) \frac{t^{n}}{n !}=\frac{2 e^{x t}}{e^{t}+1} .
$$

The numbers $\mathcal{E}_{\mathrm{n}}$, are define by $\mathcal{E}_{\mathrm{n}}:=\mathcal{E}_{\mathrm{n}}(0)$. Moreover, the polynomials $\mathcal{E}_{\mathrm{n}}(\mathrm{x})$ are Sheffer for $\left(\frac{e^{t}+1}{2}, t\right)$.

From a similar argument as in above theorem we get the following theorem.

Theorem 9 For $\mathrm{n} \geq 0$ we have

$$
\mathcal{E}_{\mathrm{n}}(\mathrm{x})=\sum_{\mathrm{k}=0}^{\mathrm{n}} \sum_{\ell=\mathrm{k}}^{\mathrm{n}}\left(\begin{array}{l}
\mathrm{n} \\
\ell
\end{array}\right) \mathcal{E}_{\mathrm{n}-\ell} \mathcal{w}_{\mathrm{m}, \mathrm{r}}(\ell, k) \mathcal{D}_{\mathrm{k}}^{[\mathrm{m}, \mathrm{r}]}(\mathrm{x}) .
$$

In the following theorem we analyze the connecting coefficients

$$
\mathcal{D}_{\mathrm{n}}^{[\mathrm{m}, \mathrm{r}]}(x)=\sum_{k=0}^{n} a_{\mathrm{n}, \mathrm{k}} \mathcal{B}_{k}(x) .
$$

Theorem 10 For $\mathrm{n} \geq 0$ we have

$$
\begin{aligned}
& \mathcal{D}_{n}^{[\mathrm{m}, \mathrm{r}]}(x)= \\
& \frac{1}{n+1} \sum_{k=0}^{n} \sum_{\ell=0}^{n-k} \sum_{s=0}^{\ell}\left(\begin{array}{c}
n+1 \\
\ell+1
\end{array}\right)\left(\begin{array}{l}
\ell+1 \\
s+1
\end{array}\right) W_{m, r}(n-\ell, k) m^{\ell-s} T_{s+1}^{[m]}(1) \mathcal{B}_{\ell-s} \mathcal{B}_{k}(x) .
\end{aligned}
$$


Proof. From Equations (24), (1) and (15) we get

$$
\begin{aligned}
a_{n, k}= & \frac{1}{k !}\left[t^{n}\right]\left(\frac{e^{\frac{e^{m}-1}{m}-1}}{\frac{e^{m t}-1}{m}} e^{r t}\left(\frac{e^{m t}-1}{m}\right)^{k}\right) \\
= & {\left[t^{n}\right]\left(\frac{e^{r t}}{k !}\left(\frac{e^{m t}-1}{m}\right)^{k} \frac{e^{\frac{e^{m}-1}{m}-1}}{t} \frac{m t}{e^{m t-1}}\right) } \\
= & {\left[t^{n}\right]\left(\left(\sum_{n=k}^{\infty} W_{m, r}(n, k) \frac{t^{n}}{n !}\right)\left(\sum_{n=0}^{\infty} T_{n}^{[m]}(1) \frac{t^{n}}{(n+1) !}\right)\left(\sum_{n=0}^{\infty} m^{n} \mathcal{B}_{n} \frac{t^{n}}{n !}\right)\right) } \\
= & {\left[t^{n}\right]\left(\left(\sum_{n=k}^{\infty} W_{m, r}(n, k) \frac{t^{n}}{n !}\right)\left(\sum_{n=0}^{\infty} \sum_{s=0}^{\ell}\left(\begin{array}{l}
\ell \\
s
\end{array}\right) m^{\ell-s} \mathcal{B}_{\ell-s} \frac{T_{s+1}^{[m]}(1)}{s+1} \frac{t^{n}}{n !}\right)\right) } \\
= & {\left[t^{n}\right]\left(\sum_{n=0}^{\infty}\left(\sum_{\ell=0}^{n-k} \sum_{s=0}^{\ell}\left(\begin{array}{l}
n \\
\ell
\end{array}\right)\left(\begin{array}{l}
\ell \\
s
\end{array}\right) W_{m, r}(n-\ell, k) m^{\ell-s} \mathcal{B}_{\ell-s} \frac{T_{s+1}^{[m]}(1)}{s+1}\right) \frac{t^{n}}{n !}\right) } \\
= & {\left[t^{n}\right]\left(\frac { 1 } { n + 1 } \sum _ { n = 0 } ^ { \infty } \left(\sum_{\ell=0}^{n-k} \sum_{s=0}^{\ell}\left(\begin{array}{l}
n+1 \\
\ell+1
\end{array}\right)\left(\begin{array}{l}
\ell+1 \\
s+1
\end{array}\right)\right.\right.} \\
& \left.\left.W_{m, r}(n-\ell, k) m^{\ell-s} \mathcal{B}_{\ell-s} T_{s+1}^{[m]}(1)\right) \frac{t^{n}}{n !}\right)
\end{aligned}
$$

Therefore, it is clear (26).

From a similar argument we get the following theorem.

Theorem 11 For $\mathrm{n} \geq 0$ we have

$$
\mathcal{D}_{n}^{[m, r]}(x)=\sum_{k=0}^{n}\left(\frac{1}{2} \sum_{\ell=0}^{n-k}\left(\begin{array}{l}
n \\
\ell
\end{array}\right) W_{m, r}(n-\ell, k) T_{\ell}^{[m]}(1)+\frac{1}{2} W_{m, r}(n, k)\right) \mathcal{E}_{k}(x) .
$$

\subsection{Some recurrence relations}

The entries in a Riordan array can be expressed as linear combination of the elements in the preceding row. That is, if $d_{n+1, k+1}$ is the $(n+1, k+1)$-th entry in a Riordan array, then there is a sequence $A=\left(a_{n}\right)_{n \geq 0}$ such that

$$
d_{n+1, k+1}=a_{0} d_{n, k}+a_{1} d_{n, k+1}+a_{2} d_{n, k+2}+\cdots, \quad a_{0} \neq 0
$$

The sequence $A$ is called the $A$-sequence [30]. Additionally, any element in column 0 , except the element $\mathrm{d}_{0,0}$, can be expressed as

$$
\mathrm{d}_{\mathrm{n}+1,0}=z_{0} \mathrm{~d}_{\mathrm{n}, 0}+z_{1} \mathrm{~d}_{\mathrm{n}, 1}+z_{2} \mathrm{~d}_{\mathrm{n}, 2}+\cdots
$$


The sequence $Z=\left(z_{n}\right)_{n \geq 0}$ is called the $Z$-sequence [22]. In general, the $A$ and $Z$ sequences, and the element $d_{0,0}$ completely characterize a proper Riordan array.

The above conditions can be write in terms of generating function. In particular, a triangular array $\mathcal{D}=\left[\mathrm{d}_{n, k}\right]_{n, k \in \mathbb{N}}$ is a Riordan array if and only if

$$
g(z)=\frac{g(0)}{1-z Z(g(z))} \quad \text { and } \quad f(z)=z(A(f(z))),
$$

where $A$ and $Z$ are the generating functions of the $A$-sequence and $Z$-sequence, respectively.

The generating function for the A-sequence of the exponential Riordan array of the $r$-Whitney numbers is given by

$$
\frac{t}{\bar{f}(t)}=\frac{t}{\ln (1+m t)^{1 / m}}=\frac{m t}{\ln (1+m t)}=\sum_{k=0}^{\infty} c_{k} m^{k} \frac{t^{k}}{k !},
$$

where $c_{k}$ are the Cauchy numbers of first kind. They are defined by $c_{n}=$ $\int_{0}^{1} x \underline{\mathfrak{n}} \mathrm{dx}$. See [23] for general information about Cauchy numbers. Taking in count that any exponential Riordan array $\langle g(x), f(x)\rangle=\left(d_{n, k}\right)_{n, k \geq 0}$ satisfies the recurrence relations (see [35, Corollary 5.7])

$$
\begin{aligned}
d_{n+1, k+1} & =\sum_{j=0}^{\infty} \frac{n+1}{k+1}\left(\begin{array}{c}
k+j \\
j
\end{array}\right) j ! a_{j} d_{n, k+j}, \\
d_{n, k}-\tilde{d}_{n-1, k} & =\sum_{\ell=k}^{n}\left(\begin{array}{c}
n-1 \\
\ell-1
\end{array}\right) f_{n-\ell+1} d_{\ell-1, k-1}, \\
k d_{n, k} & =\sum_{\ell=k}^{n}\left(\begin{array}{c}
n \\
\ell-1
\end{array}\right) f_{n-\ell+1} d_{\ell-1, k-1},
\end{aligned}
$$

where $\left(a_{j}\right)$ is the $A$-sequence and $\left(\tilde{d}_{n, k}\right)_{n, k \geq 0}=\left\langle g^{\prime}(x), f(x)\right\rangle$. Then we obtain the following corollary.

Corollary 4 The r-Whitney numbers of the second kind satisfy the following recurrence relations

$$
\begin{gathered}
W_{m, r}(n+1, k+1)=\sum_{j=0}^{\infty} \frac{n+1}{k+1}\left(\begin{array}{c}
k+j \\
j
\end{array}\right) c_{j} m^{j} W_{m, r}(n, k+j), \\
W_{m, r}(n, k)-r W_{m, r}(n-1, k)=\sum_{\ell=k}^{n}\left(\begin{array}{c}
n-1 \\
\ell-1
\end{array}\right) m^{n-\ell} W_{m, r}(\ell-1, k-1), n \geq 1,
\end{gathered}
$$




$$
k W_{m, r}(n, k)=\sum_{\ell=k}^{n}\left(\begin{array}{c}
n \\
\ell-1
\end{array}\right) m^{n-\ell} W_{m, r}(\ell-1, k-1)
$$

From the generating function (25) we obtain that the $r$-Whitney numbers of the first kind are given by the exponential Riordan array

$$
W_{1}:=\left[w_{m, r}(n, k)\right]=\left\langle(1+m z)^{-r / m}, \ln (1+m z)^{1 / m}\right\rangle .
$$

In this case, the generating function for the A-sequence of the exponential Riordan array $W_{1}$ is given by

$$
\frac{t}{\bar{f}(t)}=\frac{t}{\frac{e^{m t}-1}{m}}=\frac{m t}{e^{m t}-1}=\sum_{k=0}^{\infty} \mathcal{B}_{k} m^{k} \frac{t^{k}}{k !},
$$

where $\mathcal{B}_{\mathfrak{n}}$ are the Bernoulli numbers.

Therefore we obtain the following corollary.

Corollary $\mathbf{5}$ The $\mathbf{r}$-Whitney numbers of the first kind satisfy the following recurrence relations

$$
\begin{aligned}
& w_{m, r}(n+1, k+1)=\sum_{j=0}^{\infty} \frac{n+1}{k+1}\left(\begin{array}{c}
k+j \\
j
\end{array}\right) \mathcal{B}_{j} m^{j} w_{m, r}(n, k+j), \\
& w_{m, r}(n, k)+r \sum_{\ell=0}^{n-1}\left(\begin{array}{c}
n-1 \\
\ell
\end{array}\right)(n-\ell-1) ! w_{m, r}(\ell, k)(-m)^{n-\ell-1} \\
&=\sum_{\ell=k}^{n}\left(\begin{array}{c}
n-1 \\
\ell-1
\end{array}\right)(-m)^{n-\ell}(n-\ell) ! w_{m, r}(\ell-1, k-1), n \geq 1, \\
& k w_{m, r}(n, k)=\sum_{\ell=k}^{n}\left(\begin{array}{c}
n \\
\ell-1
\end{array}\right)(-m)^{n-\ell}(n-\ell) ! w_{m, r}(\ell-1, k-1) .
\end{aligned}
$$

\subsection{Determinantal identity}

The $r$-Whitney numbers of both kinds satisfy the following orthogonality relation (cf. [24]):

$$
\sum_{i=s}^{n} W_{m, r}(n, i) w_{m, r}(i, s)=\sum_{i=s}^{n} w_{m, r}(n, i) W_{m, r}(i, s)=\delta_{s, n},
$$


where $\delta_{s, n}=1$ if $s=n$ and 0 , otherwise. From above relations we obtain the inverse relation:

$$
f_{n}=\sum_{s=0}^{n} w_{m, r}(n, s) g_{s} \Longleftrightarrow g_{n}=\sum_{s=0}^{n} W_{m, r}(n, s) f_{s} .
$$

Moreover, we have the identity $W_{1}=W_{2}^{-1}$, where $W_{2}$ is the exponential Riordan array for the $r$-Whitney numbers of the second kind.

From definition of the $\mathrm{r}$-Dowling polynomials we obtain the equality $\mathrm{W}_{2} \cdot \mathrm{X}=$ $D_{m, r}$, where $X=\left[1, x, x^{2}, \ldots\right]^{\top}$ and $D_{m, r}=\left[\mathcal{D}_{0}^{[m, r]}(x), \mathcal{D}_{1}^{[m, r]}(x), \mathcal{D}_{2}^{[m, r]}(x), \ldots\right]^{\top}$. Then $X=W_{1} D_{m, r}$ and

$$
x^{n}=\sum_{k=0}^{n} w_{m, r}(n, k) \mathcal{D}_{k}^{[m, r]}(x) .
$$

Therefore

$$
\mathcal{D}_{n}^{[m, r]}(x)=x^{n}-\sum_{k=0}^{n-1} w_{m, r}(n, k) \mathcal{D}_{k}^{[m, r]}(x), \quad n \geq 0 .
$$

From the above equation we obtain the following determinantal identity.

Theorem 12 The r-Dowling polynomials polynomials satisfy

$$
\mathcal{D}_{n}^{[\mathrm{m}, \mathrm{r}]}(x)=(-1)^{\mathrm{n}}\left|\begin{array}{ccccc}
1 & x & \cdots & x^{\mathrm{n}-1} & x^{\mathrm{n}} \\
1 & w_{\mathrm{m}, \mathrm{r}}(1,0) & \ldots & w_{\mathrm{m}, \mathrm{r}}(\mathrm{n}-1,0) & w_{\mathrm{m}, \mathrm{r}}(\mathrm{n}, 0) \\
0 & 1 & \ldots & w_{\mathrm{m}, \mathrm{r}}(\mathrm{n}-1,1) & w_{\mathrm{m}, \mathrm{r}}(\mathrm{n}, 1) \\
\vdots & & \ldots & & \vdots \\
0 & 0 & \cdots & 1 & w_{\mathrm{m}, \mathrm{r}}(\mathrm{n}, \mathrm{n}-1)
\end{array}\right|
$$

Proof. This identity follows from Equation (28) and by expanding the determinant by the last column.

\section{Acknowledgements}

The research of the first author was supported by Universidad Antonio Nariño, Project No. 2017221 from the CVTY. The research of the second author was partially supported by Universidad Nacional de Colombia, Project No. 37805. 


\section{References}

[1] M. Benoumhani, On some numbers related to Whitney numbers of Dowling lattices, Adv. Appl. Math., 19 (1997), 106-116.

[2] M. Benoumhani, On Whitney numbers of Dowling lattices, Discrete Math., 159 (1996), 13-33.

[3] F. Bergeron, G. Labelle, P. Leroux, Combinatorial Species and Tree-Like Structures, Encyclopedia of Mathematic and its Applications, Volume 67, Cambridge University Press, Cambridge, 1998.

[4] A. Z. Broder, The r-Stirling numbers, Discrete Math., 49 (1984), 241-259.

[5] D. Callan, S.-M. Ma, T. Mansour, Some combinatorial arrays related to the Lotka-Volterra system, Electron. J. Combin., 22 (2) (2015), \# P2.22.

[6] W. Y. C. Chen. Context-Free grammars, differential operators and formal power series, Theoret. Comput. Sci., 117 (1993), 113-129.

[7] W. Y. C. Chen, A. M. Fu, Context-free grammars for permutations and increasing trees, Adv. Appl. Math., 82 (2017), 58-82.

[8] G.-S. Cheon, J.-H. Jung, r-Whitney numbers of Dowling lattices, Discrete Math., 312 (15) (2012), 2337-2348.

[9] C. B. Corcino, R. B. Corcino, R. J. Gasparin, Equivalent asymptotic formulas of second kind r-Whitney numbers, Integral Transforms Spec. Funct., 26(3)(2015), 192-202.

[10] C. B. Corcino, R. B. Corcino, I. Mezö, J. L. Ramírez, Some polynomials associated with the r-Whitney numbers, Proc. Indian Acad. Sci. Math. Sci., 128 (2018), 1-25.

[11] T. A. Dowling, A class of geometric lattices based on finite groups, $J$. Combin. Theory Ser. B, 14 (1973) 61-86. erratum; J. Combin. Theory Ser. B 15 (1973), 211.

[12] E. Gyimesi, G. Nyul, A comprehensive study of $r$-Dowling polynomials, Aequationes Math., 92 (3) (2018), 515-527.

[13] R. Hao, L. Wang, H. Yang, Context-free grammars for triangular arrays, Acta Math. Sin. (Engl. Ser.), 31 (3) (2015), 445-455. 
[14] O. Herscovici, T. Mansour, Identities involving Touchard polynomials derived from umbral calculus, Adv. Stud. Contemp. Math., (Kyungshang) 25 (1) (2015), 39-46.

[15] A. Joyal, Une théorie combinatoire des séries formelles, Adv. Math., 42 (1981), 1-82.

[16] P. Leroux, G. X. Viennot, Combinatorial resolution of systems of differential equations, I. Ordinary differential equations. In Combinatoire Enumérativé (1986), G. Labelle, P. Leroux, Eds., no. 1234 in Lecture Notes in Mathematics, Springer-Verlag, 210-245.

[17] S.-M. Ma, Some combinatorial arrays generated by context-free grammars, European J. Combin., 34 (2013), 1081-1091.

[18] T. Mansour, J. L. Ramírez, M. Shattuck, A generalization of the rWhitney numbers of the second kind, J. Comb., 8 (1) (2017) 29-55.

[19] M. Méndez, Combinatorial differential operators in: Faá di Bruno formula, enumeration of ballot paths, enriched rooted trees and increasing rooted trees. ArXiv preprint, available online at http://arxiv.org/pdf/1610.03602v1.pdf

[20] M. Merca. A convolution for the complete and elementary symmetric functions, Aequationes Math., 86 (3) (2013), 217-229.

[21] M. Merca, A note on the r-Whitney numbers of Dowling lattices, $C$. R. Math. Acad. Sci. Paris., 351 (16-17) (2013), 649-655.

[22] D. Merlini, D. G. Rogers, R. Sprugnoli, M. Cecilia Verri, On some alternative characterizations of Riordan arrays, Canadian J. Math., 49 (1997), 301-320.

[23] D. Merlini, R. Sprugnoli, M. C. Verri, The Cauchy numbers, Discrete Math., 306 (2006), 1906-1920.

[24] I. Mező, A new formula for the Bernoulli polynomials, Result. Math., 58 (3) (2010), 329-335.

[25] I. Mező, J. L. Ramírez, The linear algebra of the r-Whitney matrices, Integral Transforms Spec. Funct., 26 (3) (2015), 213-225.

[26] I. Mezö, J. L. Ramírez, Some identities of the r-Whitney numbers, Aequationes Math., 90 (2) (2016), 393-406. 
[27] M. Mihoubi, M. Rahmani, The partial r-Bell polynomials, Afr. Mat., 28 (2017), 1167-1183.

[28] M. Mihoubi, M. Tiachachat, Some applications of the $r$-Whitney numbers, C. R. Math. Acad. Sci. Paris., 352 (12) (2014), 965-969.

[29] J. L. Ramírez, M. Shattuck, Generalized r-Whitney numbers of the first kind, Ann. Math. Inform., 46 (2016), 175-193.

[30] D. G. Rogers, Pascal triangles, Catalan numbers and renewal arrays, Discrete Math., 22 (1978), 301-310.

[31] S. Roman, The Umbral Calculus, Pure and Applied Mathematics, 111. Academic Press Inc. (1984).

[32] S. Roman, G.-C. Rota,The Umbral Calculus, Adv. Math., 27 (1978), 95188.

[33] L. W. Shapiro, S. Getu, W. Woan, L. Woodson, The Riordan group, Discrete Appl. Math., 34 (1991), 229-239.

[34] M. Z. Spivey, A generalized recurrence for Bell numbers, J. Integer Seq., 11 (2008), Article 08.2.5.

[35] W. Wang, T. Wang, Generalized Riordan arrays, Discrete Math., 308 (2008), 6466-6500.

[36] A. Xu, Extensions of Spivey's Bell number formula, Electron. J. Combin., 19 (2), \#P6(2012).

[37] A. Xu, T. Zhou, Some identities related to the $r$-Whitney numbers, Integral Transforms Spec. Funct., 27 (11) (2016), 920-929.

Received: August 24, 2018 